\title{
Fabrication of an Absorber-Coupled MKID Detector and Readout for Sub-Millimeter and Far-Infrared Astronomy
}

\author{
Ari-David Brown ${ }^{\mathrm{*}, \mathrm{b}}$, Wen-Ting Hsieh ${ }^{\mathrm{a}}$ S. Harvey Moseley ${ }^{\mathrm{a}}$, Thomas R. Stevenson ${ }^{\mathrm{a}}$, \\ Kongpop U-yen ${ }^{\mathrm{a}}$, and Edward J. Wollack ${ }^{\mathrm{a}}$ \\ ${ }^{a}$ NASA Goddard Space Flight Center, Greenbelt, MD, USA 20771; \\ ${ }^{\mathrm{b}}$ MEI Technologies, Inc.,7404 Executive Place, Lanham, MD, USA 20706
}

\begin{abstract}
We have fabricated absorber-coupled microwave kinetic inductance detector (MKID) arrays for sub-millimeter and farinfrared astronomy. These sensors are comprised of arrays of $\lambda / 2$ stepped microwave impedance resonators patterned on a $1.5 \mu \mathrm{m}$ thick silicon membrane, which are optimized for optical coupling. The detector elements are supported on a $380 \mu \mathrm{m}$ thick micro-machined silicon wafer. The resonators consist of parallel plate aluminum transmission lines coupled to low impedance $\mathrm{Nb}$ microstrip traces of variable length, which set the resonant frequency of each resonator. This allows for multiplexed microwave readout and, consequently, good spatial discrimination between pixels in the array. The Al transmission lines simultaneously act to absorb optical power and by employing an appropriate surface impedance and effective filling fraction. Our novel fabrication techniques demonstrate high fabrication yield of MKID arrays on large single crystal membranes and sub-micron front-to-back alignment of the microstrip circuit.
\end{abstract}

Keywords: MKID, far-infrared, microwave, cryogenic detectors, absorber coupled

\section{INTRODUCTION}

We are developing MKID arrays for observations in the 38-400 $\mu \mathrm{m}$ spectral range. An MKID is a detector that operates upon the principle that a superconducting material's kinetic inductance and surface resistance will change in response to being exposed to radiation with a power density sufficient to break its Cooper pairs [1]. When integrated as part of a resonant circuit, the change in surface impedance will result in a shift in its resonance frequency and a decrease of its quality factor. In our approach, incident power creates quasiparticles inside a superconducting resonator, which is configured to match the impedance of free space in order to absorb the radiation being detected. For this reason MKIDs are attractive for use in large-format focal plane arrays, because they are easily multiplexed in the frequency domain and their fabrication is straightforward - which is presented here.

The salient aspects of our MKID arrays are (1) they are fabricated on single crystal silicon and (2) they are absorber coupled. We fabricated our arrays on single crystal silicon in order to reduce the noise arising from fluctuations in atomic tunneling, which are known as two-level systems and are much more prevalent in amorphous materials than in crystals [2]. The fact that our MKIDs are absorber coupled is noteworthy, because it allows for detection of far-infrared power with frequency above the energy-gap restricted cutoff of superconductor transmission lines that are commonly used [3] to couple power from an antenna to the MKID.

Our current MKIDs, which are depicted in Figure 1, are comprised of aluminum parallel plate transmission line resonators on a $1.5 \mu \mathrm{m}$ thick Si membrane. The resonators are designed so that the aluminum filling fraction yields an effective surface impedance $\sim 157 \mathrm{Ohms}$ per square, which enables frequency-independent absorption of $50 \%$ of the incident radiation. Existing provisions within this design configuration allow for the use of a quarter wave backshort to achieve near unity absorption at a fixed $\sim 30 \%$ waveband. The aluminum superconducting gap $\Delta \sim 3.5 / 2 * k_{b} T_{c}$ sets the lower bound of the detected frequency range. The diffraction limit, which is defined by the resonator geometry, sets the upper bound of efficient absorption for a free-standing structure. The resonator resonances are set by low impedance $\mathrm{Nb}$ microstrip of varying length, and these are capacitively coupled to a single $\mathrm{Nb}$ microstrip transmission line.

*ari.d.brown@nasa.gov; phone 1301 286-2293; fax 1301 286-1672

Millimeter, Submillimeter, and Far-Infrared Detectors and Instrumentation for Astronomy V, edited by Wayne S. Holland, Jonas Zmuidzinas, Proc. of SPIE Vol. 7741, 77410P

(c) 2010 SPIE · CCC code: 0277-786X/10/\$18 · doi: 10.1117/12.857281

Proc. of SPIE Vol. $774177410 \mathrm{P}-1$ 
This transmission line links all of the detector elements in the array together, and we have fabricated arrays consisting of $3 \times 3$ and 10x10 elements. Each end of the microstrip transmission line transitions into a single set of coplanar waveguide microwave launchers or input and output ports, which minimizes the readout footprint.

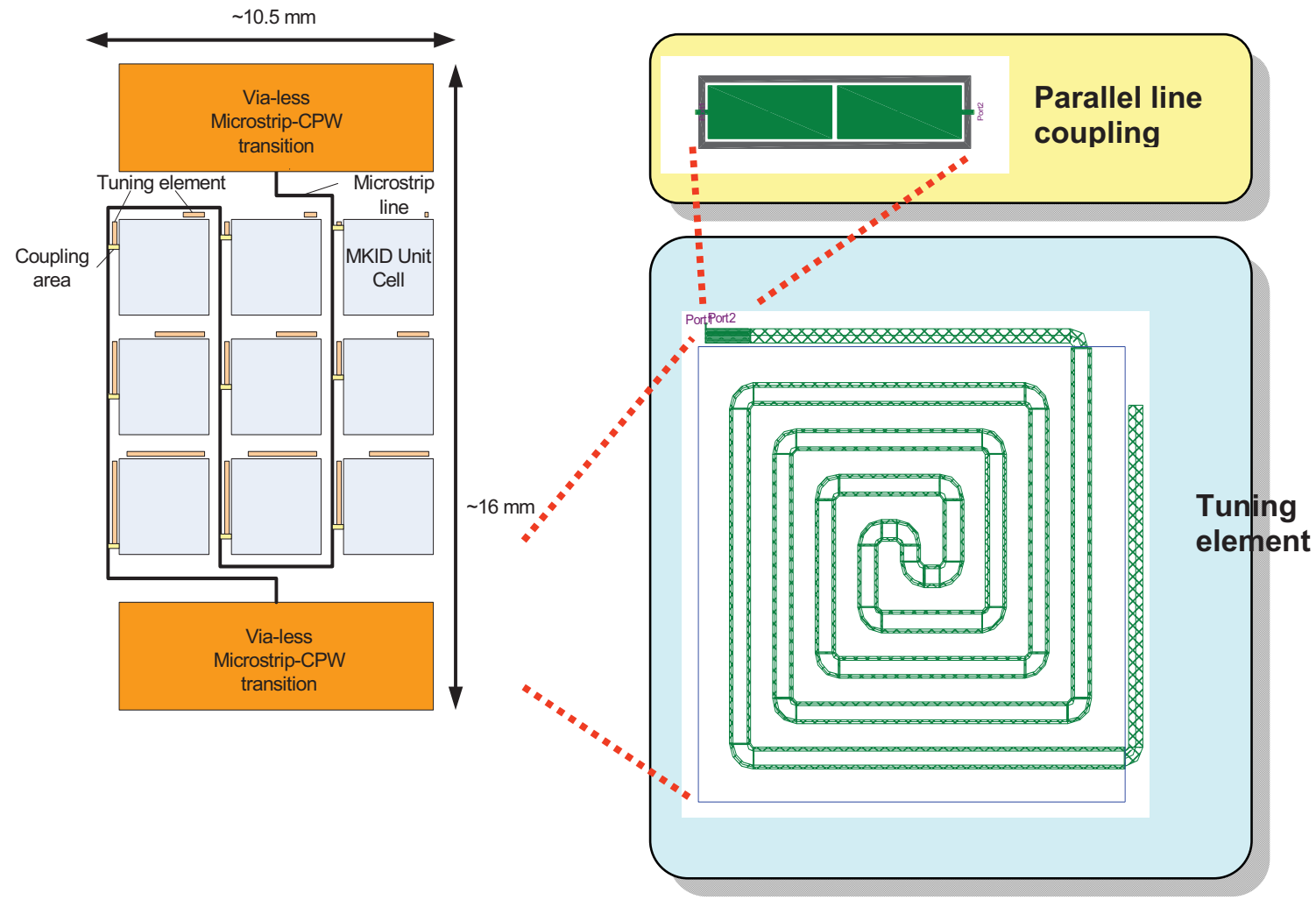

Figure 1. 3x3 absorber coupled MKID array and readout. The MKID unit cell consists of an aluminum parallel plate transmission line spiral resonator with low impedance niobium tuning elements of variable length. The resonator simultaneously acts as a broadband absorber. Each resonator is capacitively coupled to a common microstrip transmission line (top). A via-less microstrip to coplanar waveguide (CPW) allows for convenient launching of the microwave input and output.

With the exception of the microwave launchers and a portion of the microstrip transmission line, the MKID arrays are membrane suspended. We demonstrate a novel methodology that enables fabrication of silicon membranes exceeding 10 $\mathrm{mm} \times 10 \mathrm{~mm}$ in area. Furthermore, because this design implementation utilizes both microstrip and parallel plate transmission lines, metal is deposited and patterned on both sides of a silicon membrane. The use of coplanar resonators with lower field confinement and potentially reduced inter-pixel isolation would avoid this fabrication requirement. Thus, we present a straightforward means by which patterning of both sides of a silicon membrane can be accomplished in which no front-to-back alignment is required.

\section{MEMBRANE AND ALUMINUM CHARACTERIZATION}

\subsection{Mechanical model}

We fabricated mechanical models of the MKID chips prior to fabrication of the actual devices. These models consisted of $1.5 \mu \mathrm{m}$ thick silicon membranes epoxied to square and rectangular $380 \mu \mathrm{m}$ thick silicon "frames" with frame aperture dimension ranging between $4.4 \mathrm{~mm} \times 4.4 \mathrm{~mm}$ and $14.3 \mathrm{~mm} \times 14.3 \mathrm{~mm}$. The purpose of the mechanical models was to determine the maximum size of the suspended membrane on which the MKID arrays could be fabricated. 
A synopsis of the mechanical model fabrication process is presented and depicted in Figure 2. A silicon on insulator (SOI) wafer with handle wafer thickness $=400 \mu \mathrm{m}$, a buried oxide $(\mathrm{BOX})$ layer thickness $=0.4 \mu \mathrm{m}$, and silicon device

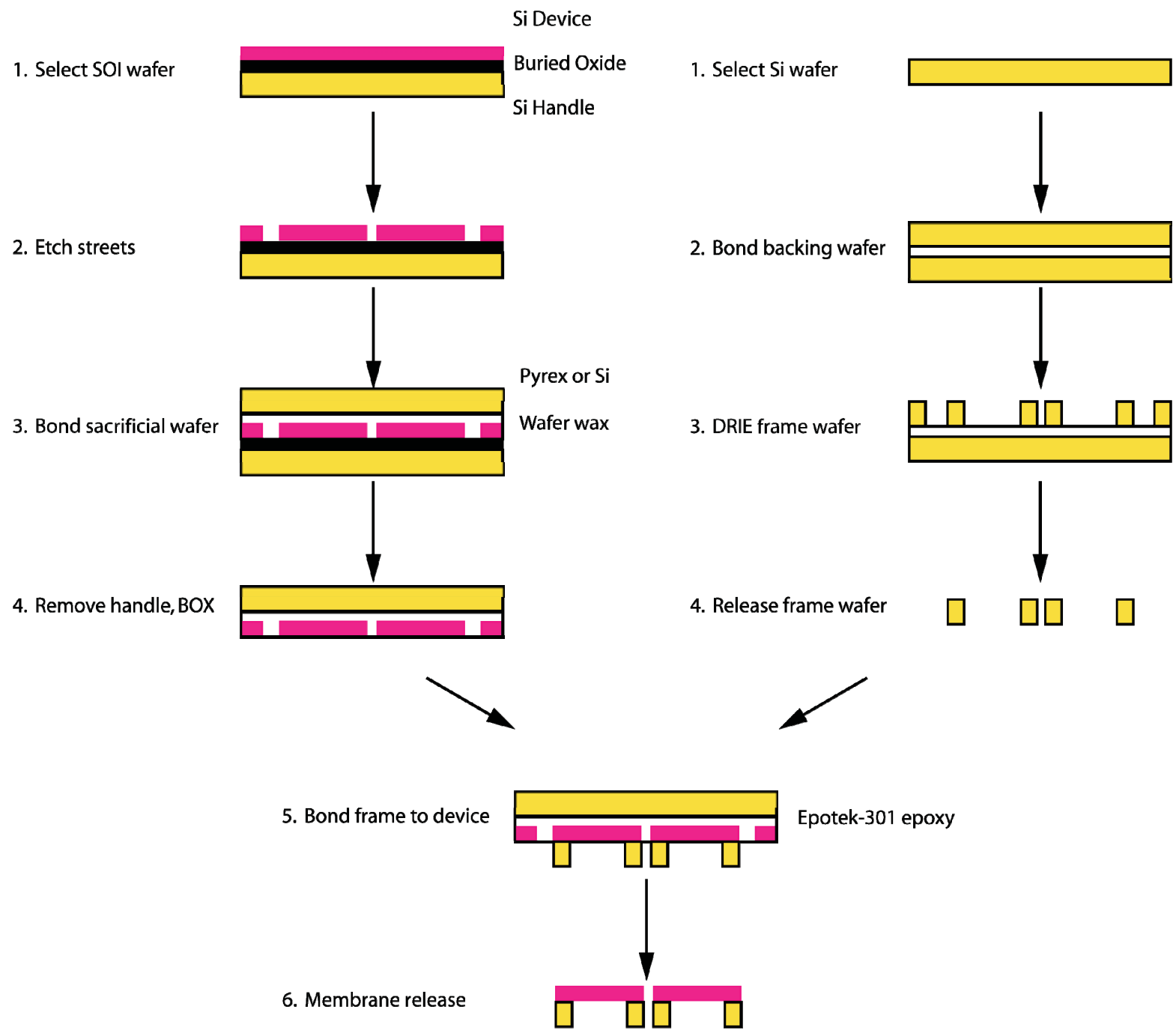

Figure 2. Mechanical model fabrication flow. The fabrication involves processing two separate, an SOI and a double-side polished, silicon wafers in parallel. The membrane areas are defined in the SOI device layer by a streets etch process (step 2) and sacrificial wafer is bonded to the membranes with a temporary bonding material in order to provide mechanical support so as to facilitate membrane handling during subsequent processing (step 3). The frame areas are defined by deep reactive ion etching of the silicon. Once the membranes have been defined and the frames have been fabricated, they are bonded together with Epotek-301 epoxy, which is a permanent bonding material.

layer thickness $=1.5 \mu \mathrm{m}$ was cleaned using a standard process. This consisted of RCA 1 (1:1:40 $\mathrm{H}_{2} \mathrm{O}_{2}: \mathrm{NH}_{4} \mathrm{OH}: \mathrm{H}_{2} \mathrm{O}$ at $75 \mathrm{C})$ and RCA $2\left(1: 1: 5 \mathrm{H}_{2} \mathrm{O}_{2}: \mathrm{HCl}: \mathrm{H}_{2} \mathrm{O}\right.$ at $\left.75 \mathrm{C}\right)$ cleans, and a 1:10 dilution of hydrofluoric acid in water dip to remove the native oxide layer. Cut-outs, which were defined by narrow trenches or "streets" around the perimeter of the cut-out, in the device layer were patterned with Shipley-1827 photoresist and etched with an $\mathrm{SF}_{6} / \mathrm{O}_{2}$ plasma inside a reactive ion etcher (RIE). The wafer was then bonded to a Pyrex wafer, coated with Crystalbond-509 wafer wax, using a wafer bonder (Logitech). Thinning of the silicon handle wafer was accomplished via lapping, using a Logitech PM-5, until its thickness was approximately $100 \mathrm{um}$. We subsequently used a $600 \mathrm{~W} \mathrm{SF} 6 / \mathrm{O}_{2}$ plasma generated by a deep reactive ion etcher (DRIE) (STS) to remove the remaining silicon. It is important to note that the silicon etch rate is faster at the wafer edges than at the center. Consequently, the handle wafer would be completely etched at the wafer edge prior to 
the other regions. In order to conserve the silicon device wafer at the wafer edge the DRIE platen power (i.e., the plasma power directed at the wafer) was turned off once complete removal of the handle wafer in that region occurred. In this manner, the plasma consumed the remaining exposed silicon handle layer. Nonetheless, the plasma did not etch the newly exposed oxide BOX layer because the ions were deflected by charge build-up in this insulating layer. We noted that occasionally device wafer regions near the wafer edge would become corrugated. It was believed that this arose from (1) differential thermal expansion between silicon and Pyrex and (2) localized melting of the wax. The BOX layer was subsequently dissolved in a buffered hydrofluoric acid etchant.

The silicon frame was fabricated by cleaning a 4" $\mathrm{Si}(001)$ wafer using the $\mathrm{SC} 1 / \mathrm{SC} 2$ technique described above. A Pyrex backing wafer was bonded to it using Crystalbond-509 wax. The silicon frame cut-outs were defined via streets and lithographically patterned using a thick, $12 \mu \mathrm{m}$, AZ-4620 photoresist. The streets were then etched by DRIE using the Bosch process in which highly sharp sidewall profiles can be realized. Finally, the Si frames were released from the Pyrex backing wafer in acetone.

The final step in fabricating the mechanical models involved attaching the $1.5 \mu \mathrm{m}$ thick silicon membrane to the frame. For this purpose, we employed Epotek-301 epoxy [4]. Epotek-301 is an optically transparent epoxy that has a relatively low thermal expansion coefficient $(\mathrm{TCE})=39 \times 10^{-6}$ and a shear strength $>138 \mathrm{MPa}$. These properties make it highly resilient to thermal cycling between room temperature and cryogenic temperature required for MKID operation. Furthermore, we found that Epotek-301 was insoluble in acetone. This allows for silicon membrane release from the Pyrex backing wafer in acetone - which is explained below.

The individual silicon frames were spray coated with Epotek-301 that had been diluted with toluene. Uniform coating of the sample was achieved by slowly rotating it over a range of -90 to 90 degrees in front of the spray nozzle, which we defined as one spray pass. We set the flow emerging from the spray nozzle so as to obtain a dilute epoxy density $=3.2$ $\mu \mathrm{g} / \mathrm{mm}^{2}$. The parts were then coated for a total of three passes for good epoxy uniformity. They were then outgassed in vacuum for $8 \mathrm{~min}$, followed by a $6 \mathrm{~h}$ partial cure at room temperature in atmosphere. The frames were placed onto the Pyrex-backed silicon device layer, and the combination of the two was bonded together inside a wafer bonder at $65 \mathrm{C}$ and 0.5 bar bond pressure for $1 \mathrm{~h}$. In order to release the Pyrex wafer from the silicon membrane, the wafer stack was soaked in acetone for several days. Once released, the parts were soaked in isopropanol and dried on a hotplate set to $65 \mathrm{C}$.

Optical micrographs of released mechanical models are shown in Figure 3, showing that the unsupported membrane area exceeded $14 \mathrm{~mm}$ x $14 \mathrm{~mm}$ with high yield. Consequently, this permitted that we could design our devices to have $10 \mathrm{x}$ 10 arrays of $1 \mathrm{~mm} \times 1 \mathrm{~mm}$ MKIDs and expect a high fabrication yield with a high degree of confidence.

The mechanical model also allowed us to determine whether or not there were design limitations associated with the use of Epotek-301 to epoxy the membrane to the frame wafer. One concern was that the Epotek would wet the entire membrane area during bonding. This would be deleterious for our devices, because Epotek has a fairly high loss tangent which would degrade detector performance. Fortunately, we found that Epotek had a tendency to wet the walls of the frame. Another concern was that there would be dielectric loss in the ground plane regions under the frame. We ultimately mitigated the possibility of this unwanted effect by extending the silicon membrane (i.e., Epotek-free regions) by $1 \mathrm{~mm}$ on each side around the devices.

Furthermore, the mechanical model helped us to confirm the epoxy mechanical integrity upon thermal cycling. One structure was rapidly thermally cycled by affixing it to a wooden dowel rod with floss and pulling it in and out of a liquid nitrogen-filled dewar. This process was conducted 30 times and resulted in no apparent membrane structural damage. 


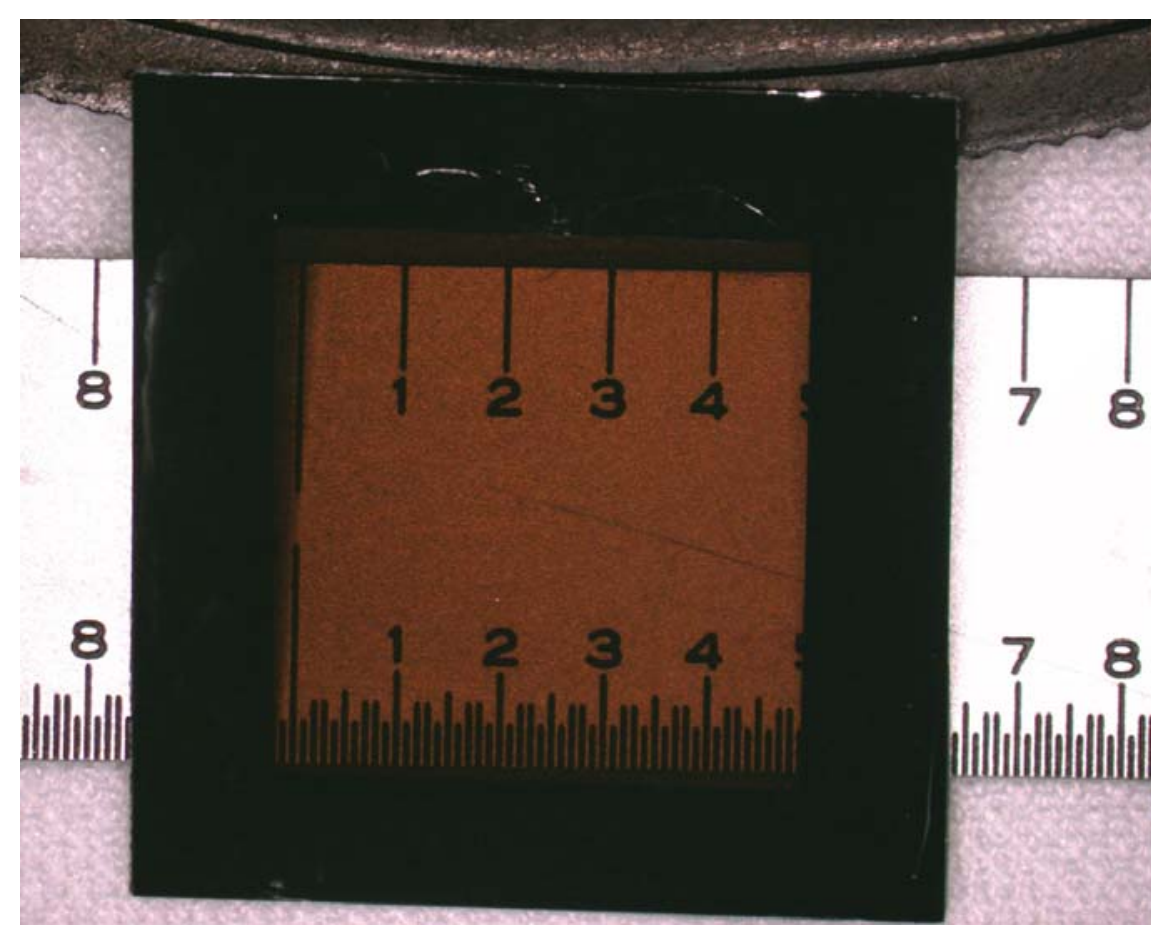

Figure 3. Image of a $14.3 \mathrm{~mm} \times 14.3 \mathrm{~mm}$ membrane mechanical model structure. The transparent region surrounded by the dark frame is the $1.5 \mu \mathrm{m}$ single crystal silicon membrane.

\subsection{Aluminum thin films}

The aluminum films, used for microwave resonators and optical coupling structure, provide primary detection functions for the MKIDs. For this reason, we developed a fabrication procedure that insured tight control over the film quality and thickness. The resonant structures consist of spiral parallel plate transmission lines and can be treated, in the long wavelength limit, as part of an effective medium in which radiation of both polarizations can be absorbed. Thus, for aluminum resonators with filling fractions of order $5-6.5 \%$, the aluminum film sheet resistance has to be approximately 7.8-10.5 Ohms per square for optimal optical coupling.

The aluminum films were electron-beam deposited inside a chamber with base pressure $\sim 10^{-7}$ Torr. In order to minimize the probability of film contamination, the chamber walls were coated with an aluminum thin film immediately prior to deposition. During this preparation run, a witness sample would be coated with aluminum and subsequently characterized before proceeding with the device wafer run. Furthermore, the wafers were initially ion milled with $\mathrm{Ar}^{+}$ ions so as to remove the native oxide layer. We then deposited the $\mathrm{Al}$ at a rate $=0.15 \mathrm{~nm} / \mathrm{sec}$. In a previous study [5] we found that $\mathrm{Al}$ thin films of order $10 \mathrm{~nm}$ thick could be made to have a $4.2 \mathrm{~K}$ sheet resistance $=10.5 \mathrm{Ohms} / \mathrm{sq}$. Here we found that at this scale, the quartz crystal deposition monitor (Inficon used for estimating the film thickness was highly non-linear and gave non-reproducible measurements for very thin films. Thus, it was decided that we would deposit the thinnest film for which the monitor was reliable, which was determined to be $17.7 \mathrm{~nm}$. This is lower than the dirty limit coherence length of several hundred $\mathrm{nm}$ and penetration depth of approximately $100 \mathrm{~nm}$. At this thickness four-wire DC measurements yielded low temperature sheet resistance, resistivity, and residual resistance ratio (RRR), which is defined here as $\mathrm{R}_{295 \mathrm{~K}} / \mathrm{R}_{4.2 \mathrm{~K}}$, equal to $5 \mathrm{Ohms}$ per square, $8.9 \mu \mathrm{Ohm} \mathrm{cm}$, and 1.4, respectively. Assuming that the mean-free path resistivity product $=3.94 \times 10^{-16} \mathrm{Ohm} \mathrm{m}^{2}$ the estimated mean-free path $=4.4 \mathrm{~nm}$, which is approximately one quarter that of the thickness. Room temperature optical transmission measurements, obtained using a Fourier transform spectrometer, are shown in Figure 4. We fit the data using a transmission line model and found that the extracted sheet resistance $=7 \mathrm{Ohms}$ per square, which is identical to the $\mathrm{DC}$ value. This is noteworthy, because the optical measurements were taken over one month after the DC ones. Thus, it appeared that sample degradation because of 
significant surface oxidation did not occur. Our $\mathrm{Al}$ films were also superconducting, with superconducting transition temperature $=1.57 \mathrm{~K}$ and a critical current density $>50 \mathrm{~A} / \mathrm{cm}^{2}$.

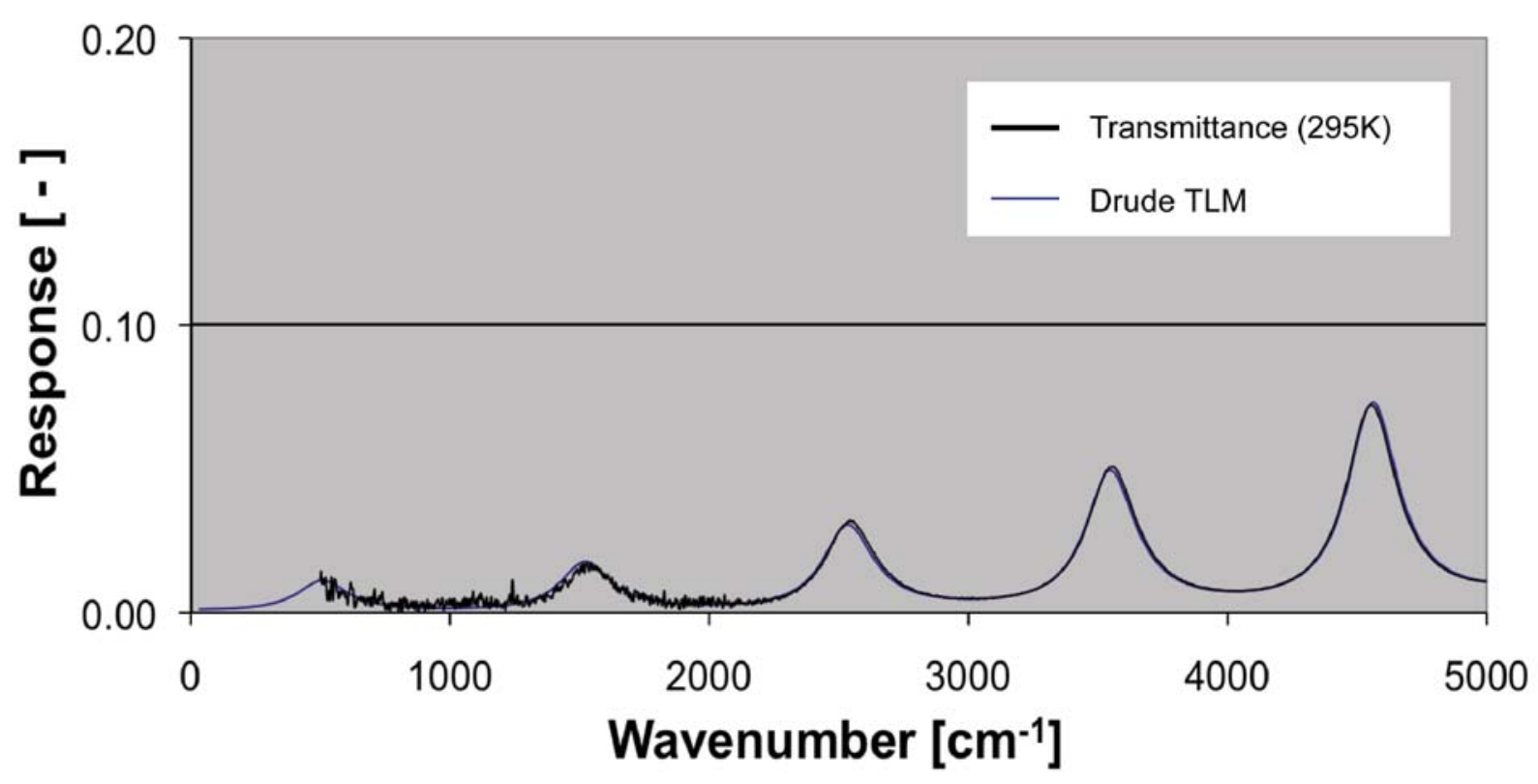

Figure 4. Optical response of $17.7 \mathrm{~nm} \mathrm{Al}$ thin film on a $1.42 \mu \mathrm{m} \mathrm{Si} \mathrm{membrane.} \mathrm{The} \mathrm{data} \mathrm{were} \mathrm{collected} \mathrm{using} \mathrm{a}$ Fourier transform spectrometer (Brucker) and are well fitted by a Drude transmission line model (TLM). The sheet impedance extracted from the model is 7 Ohms per square, which is the same value obtained via a DC electrical measurement.

\section{MKID ARRAY FABRICATION}

\subsection{Front side processing}

A cartoon of this process is depicted in Figure 5. We started the process by cleaning SOI wafers (Soitec), which had a $1.52 \mu \mathrm{m}$ thick Si device layer, with our standard SC1/SC2 solutions. Alignment marks were patterned with S-1827 photoresist and reactive ion etched in an $\mathrm{SF}_{6} / \mathrm{O}_{2}$ plasma. We etched completely through the device layer so as to avoid the need for front-to-back alignment in subsequent backside patterning. The native oxide layer was removed in an RFgenerated Ar plasma. Subsequently, an Nb/Au bilayer film was DC magnetron sputter deposited in situ, i.e., without breaking vacuum. The $\mathrm{Nb}$, which was deposited first, would serve as the microstrip transmission line as well as the low impedance portions of the resonators. We deposited $250 \mathrm{~nm}$ of the material, which is approximately three times its magnetic penetration depth. This insured that the material maintained its superconductivity in the presence of the magnetic field generated by the resonators. Only a very thin, $\sim 20 \mathrm{~nm}$, gold layer was sputter deposited. Its main purpose was to passivate the $\mathrm{Nb}$ and, consequently, prevent the formation of a Josephson junction between the $\mathrm{Nb}$ and Al. The Au was patterned with an S-1811 photomask and etched in an iodine solution (KI: $\left.\mathrm{I}_{2}: \mathrm{H}_{2} 0 ; 4: 1: 40\right)$. We intentionally over etched the $\mathrm{Au}$ in order to avoid masking the $\mathrm{Nb}$, which was patterned and etched with S-1827 photoresist and a $\mathrm{CF}_{4} / \mathrm{O}_{2}$ plasma, respectively. We controlled the sidewall angle of the $\mathrm{Nb}$ step edge by adjusting the 
flow ratio of $\mathrm{O}_{2}$ to $\mathrm{CF}_{4}$, and an angle of $\sim 45$ degrees was used. Given the fact that the fluorine chemistry also etches silicon, the control of the $\mathrm{Nb}$ etching is extremely critical. The $\mathrm{Al}$ film for the front side resonator was deposited and patterned with S-1811 photoresist. A phosphoric acid-based solution was then used to etch the Al. The final step in front side processing of our devices involved bonding the metalized side of the wafer to a Pyrex wafer with Crystalbond509 wafer wax.

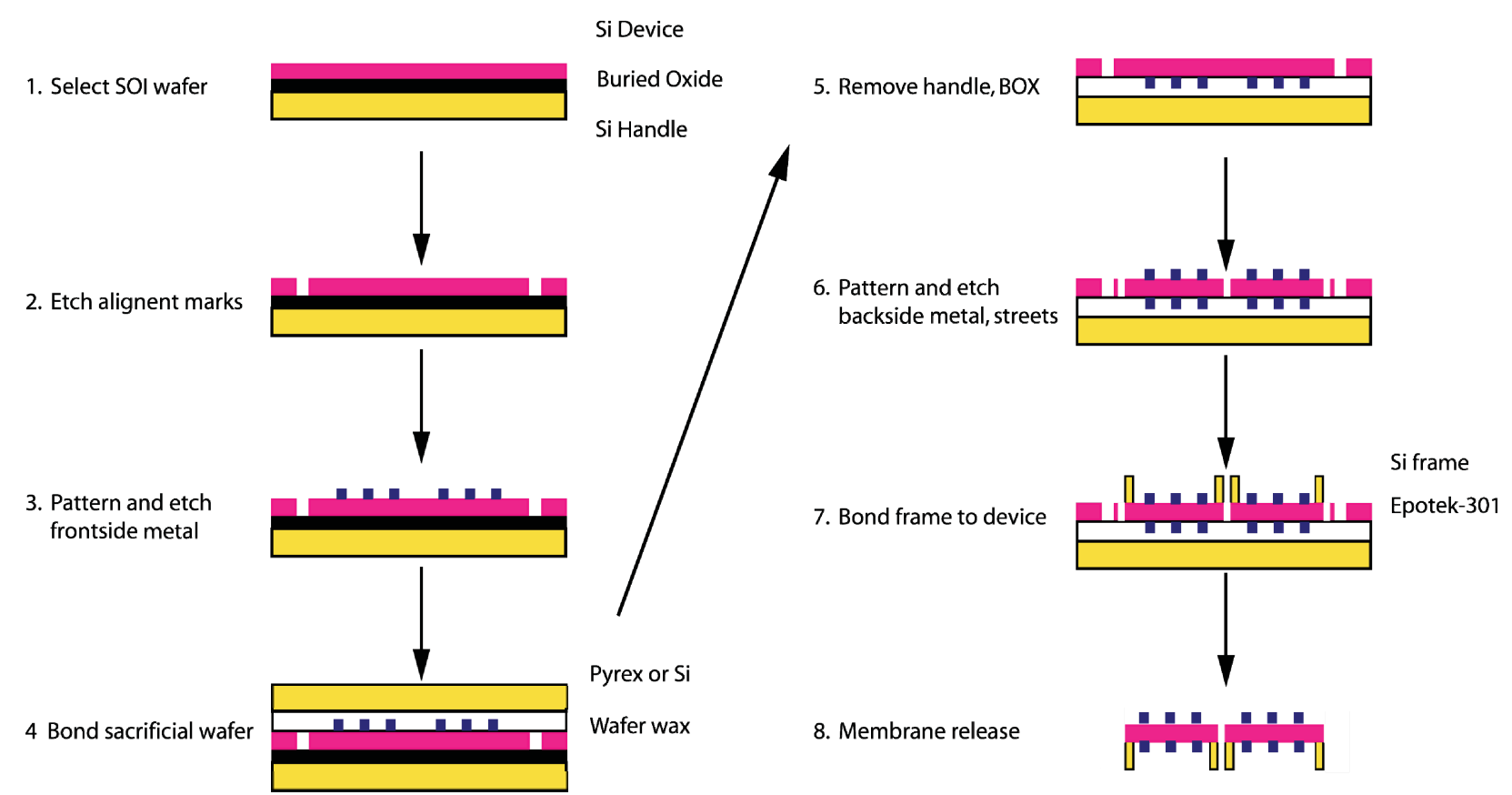

Figure 5. MKID fabrication flow. This process is very similar to the mechanical model process outlined in Figure 2 , with the addition of metallization of both sides of the silicon membrane. Of note is the alignment marks etching process (step 2), in which the alignment marks are etched all the way through the SOI silicon device layer. This permits good front-to-back alignment without having to employ backside photolithography.

\subsection{Back side processing}

This process commenced with the removal of the SOI silicon handle layer, which consisted of wafer lapping followed by a DRIE etch. An $\mathrm{Nb}$, as a superconducting groundplane, and an $\mathrm{Au}$, for $\mathrm{Nb}$ passivation, layer stack was then sputter deposited. This process elucidated the shortcomings of using Crystalbond-509 as a temporary bonding material, because the silicon device wafer became corrugated, with corrugation lengthscale of approximately one millimeter, upon sputtering of the $\mathrm{Nb} / \mathrm{Au}$. The metal stack was found to have a compressive film stress of $\sim 200 \mathrm{MPa}$ when deposited on silicon and it is believed that the steady state wafer temperature exceeded $100^{\circ} \mathrm{C}$ during sputtering. Crystalbond-509 is known to have a glass transition temperature $<75^{\circ} \mathrm{C}$. Thus, we ascribed corrugation of the silicon membrane to an instability driven by a reduction of stress in the $\mathrm{Nb} / \mathrm{Au}$, which would result in deformation of the silicon membrane.

In order to prevent further corrugation of the silicon membrane, the maximum temperature that we allowed the wafers to reach was $65 \mathrm{C}$ in subsequent processing. This condition necessitated several important modifications to our photolithography processes. For instance, we extended photoresist bake times, and, instead of performing a high temperature hard bake, a UV crosslinker (UVP) was used to crosslink the resist. In addition, because the silicon surface was corrugated, the photomask had to be positioned $50 \mu \mathrm{m}$ above the wafer during UV exposure of the photoresist. This alignment gap made it much more difficult to properly align the wafer. Consequently, we used a witness wafer in order to ascertain the magnitude of any potential misalignment resulting from the alignment gap prior to exposing our device wafers. Thus, we could manually correct this systematic alignment error and obtain very good front-to-back alignment on some of our devices, which is shown in Figure 6. 

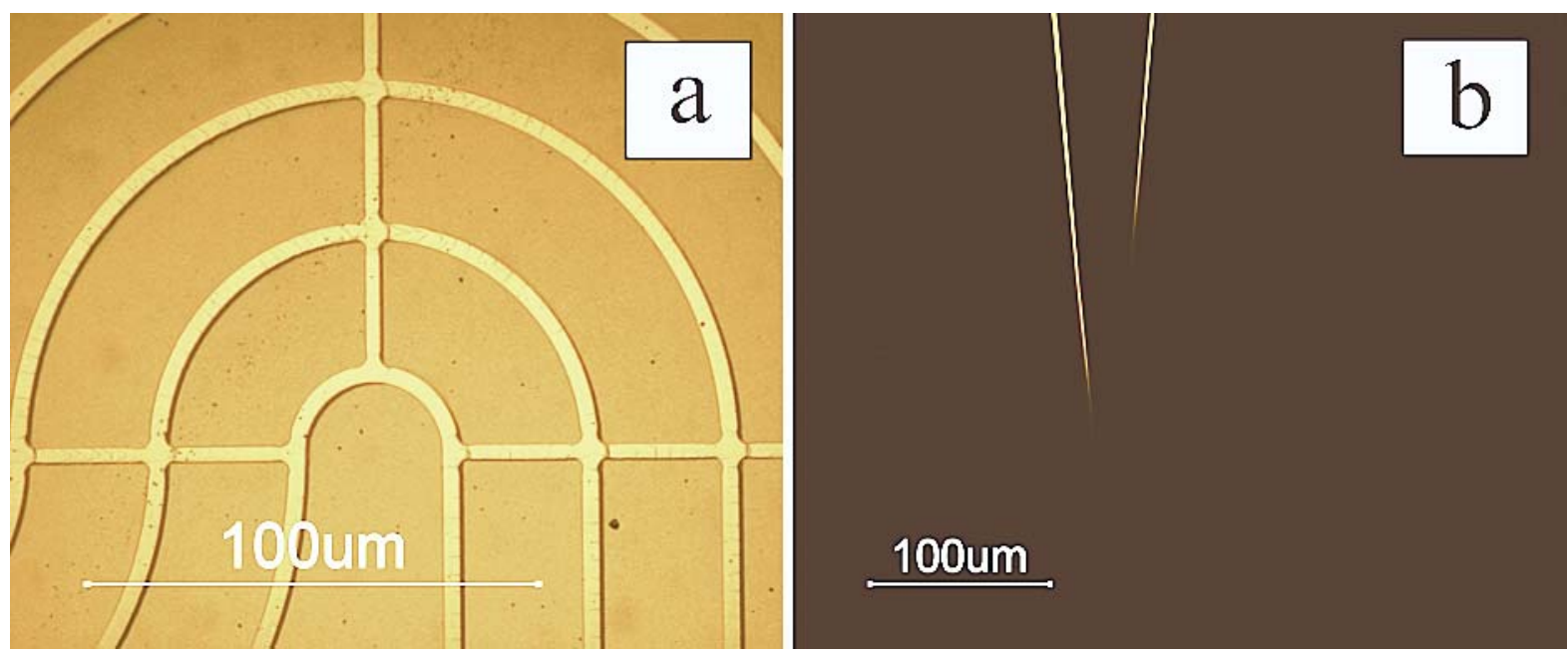

Figure 6. Micrographs of MKID features illustrating the degree of front-to-back misalignment, in which the features were illuminated on both sides of the silicon membrane. (a) Aluminum resonator, which consists of a parallel plate transmission line. The "shadow" on the features represents the degree of misalignment, which is approximately 0.4 microns. (b) Coplanar waveguide - microstrip transition. The triangular region in the center of the image should have a light-colored boundary around it. We estimated the misalignment here to be less than one micron.

After depositing the $\mathrm{Nb} / \mathrm{Au}$, the metals were patterned and etched as described in the front side processing section of the text. An aluminum film, which served as the second half of the parallel plate transmission line resonator, was also deposited and etched in a manner similar to the one described above. Streets in the silicon membrane were defined with S-1827 photoresist and etched with a $\mathrm{CF}_{4} / \mathrm{N}_{2} \mathrm{O}$ plasma, to remove the $\mathrm{Nb}$, and $\mathrm{SF}_{6} / \mathrm{O}_{2}$, to remove the $\mathrm{Si}$.

\subsection{Frame fabrication, mounting, and release}

The frame was fabricated by patterning and DRIE etching a $380 \mu$ m thick $\operatorname{Si}(001)$ wafer, with a process similar to that used to fabricate the mechanical model frames. The individual frames were spray coated with Epotek-301 and were subsequently manually aligned and bonded to the device layers. The MKID chips were released in acetone over a period of five days and were subsequently cleaned in acetone to remove Crystalbond-509 residue, toluene to remove Epotek301 residue, and isopropanol. We dried the chips on a hotplate set to $65 \mathrm{C}$.

Some images of released chips are shown in Figure 7. Our "standard" MKID chips consisted of five 3x3 MKID arrays with resonance frequency ranging between 0.763 and $2.15 \mathrm{GHz}$. We also fabricated "large" MKID chips which consisted of single 10x10 MKID arrays and "small" MKID chips with one 3x3 MKID array. The fabrication process is extremely robust, and we have $100 \%$ yield on the MKID detector fabrication in which every membrane survived the processing. One should note that, perhaps, the most important aspect of our fabrication process that needs further refinement is the front to back alignment. Because of issues related to corrugation, the front-to-back misalignment was found to vary between 0.4 and $6 \mu \mathrm{m}$ across a wafer in our initial efforts. 

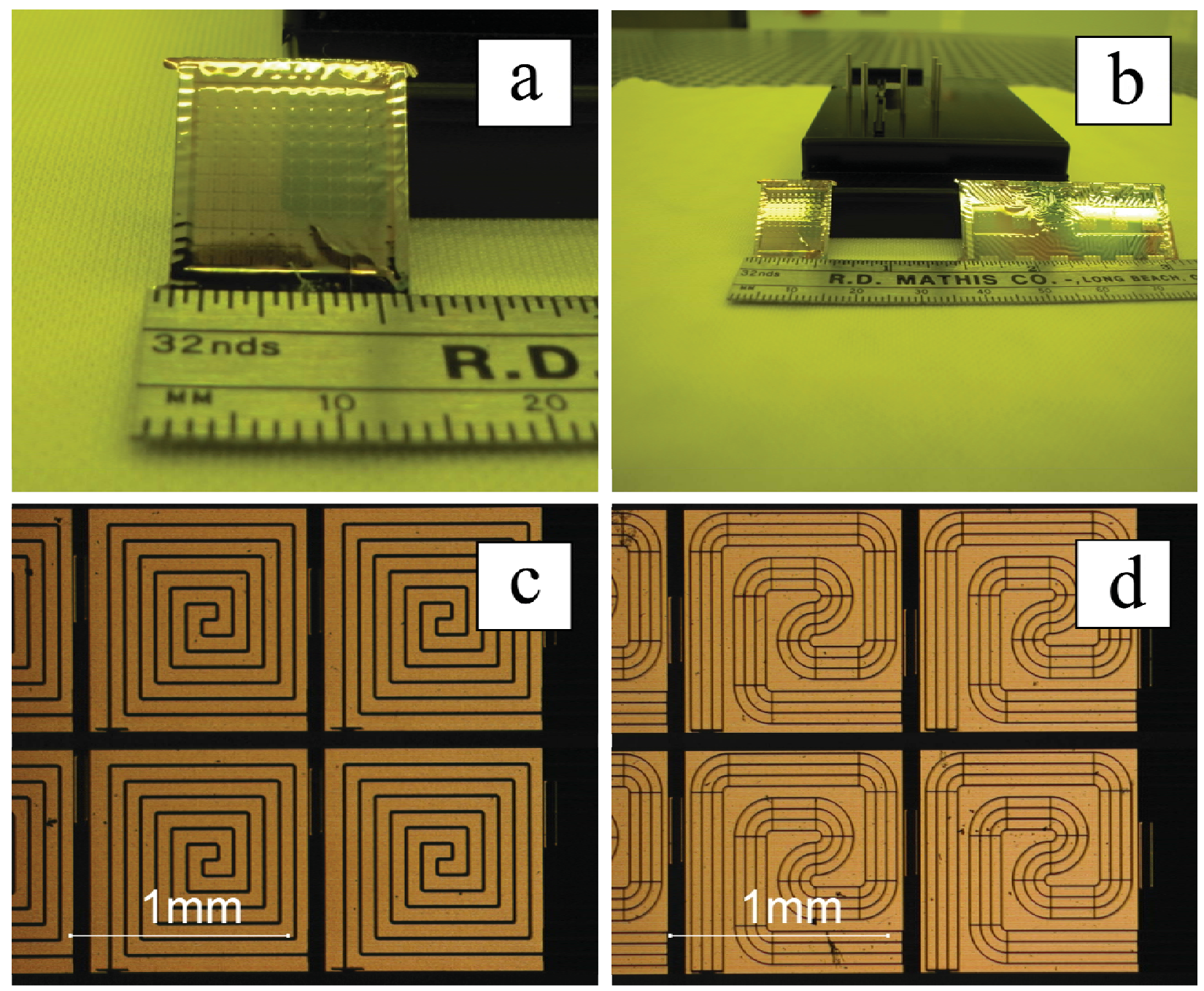

Figure 7. Images of released MKID array chips. (a) $10 \times 10$ MKID array chip. (b) $10 \times 10$ and "standard" MKID array chips. The standard chips possess five 3x3 MKID arrays. (c) and (d) Some higher magnification views of four resonators which comprise part of the $3 \times 3$ MKID arrays. The arrays are designed to have resonance in the 1.039-1.067 and 2.093-2.15 GHz frequency ranges (c) and (d), respectively.

\subsection{MKID chip packaging}

The chip was packaged inside a metal enclosure that had apertures on two faces for device illumination and three pairs of SMA ports on, two other, opposing faces. The SMA pins were wire bonded to gold $50 \mathrm{Ohm} \mathrm{CPW}$ regions electroplated on alumina boards. These regions were impedance matched to the coaxial microwave readout lines and wire bonded to the CPW launchers on the chips, and each CPW region was electromagnetically shielded from the other regions with a metal wall. Thus, three MKID arrays could potentially be tested simultaneously with minimal crosstalk. Two G-10 clips were used to mount the MKID chips so as to prevent silicon frame damage arising from CTE mismatch upon cooling. These clips proved to be somewhat inadequate in providing chip support during wirebonding. To realize the electrical connections for our preliminary testing small droplets of small droplets of Epotek- $\mathrm{H} 2 \mathrm{O}$ conductive epoxy were used to connect the device side of the $25 \mu \mathrm{m}$ gold bond wire. 


\section{ALTERNATE FABRICATION APPROACHES AND MATERIALS}

We are presently exploring other materials in order to (1) reduce or eliminate corrugation during processing, and (2) increase the MKID resonator filling fraction to improve absorption efficiency at high frequencies.

\subsection{Mitigating membrane corrugation}

In addressing the first concern, we are exploring the use of alternative temporary bonding materials for attaching the silicon membrane to a Pyrex backing wafer. Our present candidate material is CR-200 (Brewer-Science), which is a spin-on epoxy with a melting temperature $\sim 200 \mathrm{C}$. Furthermore, it dissolves readily in a non-polar solvent that does not attack Epotek-301. Thus, this epoxy is compatible with our current process and it is expected to minimize the risk of silicon membrane corrugation during processing because its melting temperature is much higher than that of Crystalbond-509.

\subsection{Using TiN as a resonator material}

In addressing the second design consideration, recipes for different resonator materials with much lower resistivity than aluminum are being explored. This will result in obtaining higher control for achieving the target sheet resistance ( 1 and $20 \mathrm{Ohms}$ per square) for the desired films. To this end, we have begun investigating the properties of TiN thin films deposited via reactive DC magnetron sputtering [6]. TiN is an attractive material for MKID applications because in addition to being almost impervious to oxidation, in bulk form its resistivity $=40 \mu \mathrm{Ohm} \mathrm{cm}$ and its $T_{\mathrm{c}}=4.7 \mathrm{~K}$. We have made films that possess resistivity ranging between $100-7000 \mu \mathrm{Ohm} \mathrm{cm}$, which is approximately 10-800 times that of $\mathrm{Al}$, and with $T_{\mathrm{c}}$ ranging between 0.17 and $4.7 \mathrm{~K}$ by simply adjusting the deposition power and ratio of $\mathrm{Ar}$ to $\mathrm{N}_{2}$ partial pressure. Furthermore, we have experimentally demonstrated TiN microwave resonators with quality factors in excess of $9 \times 10^{4}$ at $0.37 \mathrm{~K}$.

\section{CONCLUSIONS}

We have developed an MKID array fabrication process, which incorporated a novel technique in which both sides of a $1.5 \mu \mathrm{m}$ thick single crystal $\mathrm{Si}$ membrane were patterned without the use of back side photolithography. It is demonstrated that this process enables the fabrication of frequency domain multiplexed $10 \times 10$ MKID arrays designed for far-infrared astronomy. Furthermore, we show that our aluminum films possess both optical and superconducting properties that are anticipated for optimal MKID performance. Two alternate fabrication approaches are presented, which will further refine this process and, ultimately, enhance device performance.

\section{REFERENCES}

[1] P. K. Day, H. G. LeDuc, B. A. Mazin, A. Vayonakis, and J. Zmuidzinas, "A Broadband Superconducting Detector Suitable for use in Large Arrays," Nature 425, 817 (2003).

[2] J. Gao, J. Zmuidzinas, B. A. Mazin, H. G. LeDuc, and P. K. Day, "Noise Properties of Superconducting Coplanar Waveguide Structures," Appl. Phys. Lett. 90, 102507 (2007).

[3] P. K. Day, H. G. Leduc, A. Goldin, T. Vayonakis, B.A. Mazin, S. Kumar, J. Gao, and J. Zmuidzinas, "Antennacoupled Microwave Kinetic Inductance Detectors,” Nucl. Instr. And Meth. In Phys. Res. A 559, 561 (2006).

[4] www.epotek.com/SSCDocs/datasheets/301.PDF

[5] T. R. Stevenson, J.S. Adams, W.T. Hsieh, S.H. Moseley, D.E.Travers, K.U-yen, E.J. Wollack, and J. Zmuidzinas, " Superconducting Films for Absorber-Coupled MKID Detectors for Sub-Millimeter and Far-Infrared Astronomy," IEEE Trans. Appl. Supercond. 19, 561 (2009).

[6] H. G. Leduc, B. Bumble, P.K. Day, A. D. Turner, B. H. Eom, S. Golwala, D. C. Moore, O. Noroozian, J. Gao, B. A. Mazin, S. McHugh, and A. Merrill, "Titanium Nitride Films for Ultrasensitive Microresonator Detectors," arxiv.org/pdf/1003.5584 (2010). 